\title{
A process approach to improving pain management in the emergency department: development and evaluation
}

\author{
Anne-Maree Kelly
}

\begin{abstract}
Aim-(1) To describe a process approach to the improvement of pain management in emergency departments. (2) To compare analgesia ordering and administration practices for patients with acute fractures before and after implementation of a nurse managed, titrated intravenous narcotic policy.

Method-Retrospective chart review of patients with long bone fractures for the years 1993 and 1997.

Results-There was a dramatic change in analgesia administration practices away from the intramuscular route in favour of the intravenous route $(p<0.001)$. For long bone fractures, in $1993,53 \%$ of patients received intramuscular narcotic analgesia compared with $5 \%$ in 1997 . In contrast, in $1993,6 \%$ of the patients received intravenous narcotic analgesia compared with $54 \%$ in 1997.

Conclusion-This study demonstrates that a process approach to improving pain management that resulted in both changes in drug administration and pain assessment and management processes made a significant and sustained change to analgesia ordering and administration practices for patients with long bone fractures in an emergency department. (f Accid Emerg Med 2000;17:185-187)
\end{abstract}

Keywords: pain managment

Pain is a very common reason for presenting to the emergency department (ED). ${ }^{12}$ While the causes for pain are diverse, ranging from fractures and other injuries to chest or abdominal pain or headache, the provision of effective, timely analgesia should be one of the principal goals of emergency staff. Studies suggests that EDs do not perform well in this area. $^{3-7}$ The reasons identified for this include inadequate knowledge of analgesic pharmacology, the use of inadequate doses of analgesic agents given by inappropriate routes of administration and poor processes for the provision of analgesia. ${ }^{7-9}$

It has been recommended that, when narcotic agents are the analgesic drugs of choice, these should be administered intravenously (IV) for the relief of severe pain. ${ }^{10}{ }^{11} \mathrm{~A}$ pilot audit of the use of analgesic agents in the Department of Emergency Medicine at Western Hospital, Melbourne conducted in March 1994 showed that only $10 \%$ of narcotic analge- sia was being given by the IV route and that this was almost entirely confined to patients with chest pain that was suspected to be of myocardial origin. In this case, the reason for the use of the IV route seemed to be as much a reluctance to interfere with cardiac enzyme measurements as an attempt to provide rapid analgesia. In that series, a significant proportion of patients who had been treated with intramuscular (IM) analgesia continued to report severe pain more than 30 minutes after initial treatment and many required further doses of analgesia. In response to these findings, the Department of Emergency Medicine decided that improvement was necessary and a process approach was chosen to ensure that the improvement(s) identified were appropriate and practical in our practice setting.

This paper outlines the strategies for change identified by the process of conducting a detailed review of analgesia practice and reports the change in practice after implementation of these changes.

\section{Method}

THE REVIEW PROCESS AND OUTCOMES

The review was conducted by a multidisciplinary team with the aim of providing prompt, effective analgesia to all patients experiencing pain requiring parenteral therapy. Each of the stages in provision of adequate analgesia were examined: the expression of pain by patients, its recognition by health care workers, the ordering of an analgesic, the review of the effectiveness of that treatment and its augmentation if needed. The major deficiencies identified were:

(1) Inadequate and inconsistent inquiry about and documentation of patients' pain experience both at presentation and throughout their ED treatment

(2) Inadequate doses of analgesic agents being administered by inappropriate routes

(3) Delays in augmentation of inadequate analgesia and

(4) Pain management not being seen as a high priority.

The strategies implemented in response to these findings were:

(1) Routine patient reporting of pain as part of "normal" nursing observations

(2) A change in the culture of the ED recognising that pain management is a high priority for patients

(3) A decision to move to titrated IV opioids as the preferred method of administration of narcotic analgesia. 
Table 1 Types of fractures sustained by the study groups

\begin{tabular}{lll}
\hline Type of fracture & $\begin{array}{l}\text { Number subjects } \\
1993\end{array}$ & $\begin{array}{l}\text { Number patients } \\
1997\end{array}$ \\
\hline Femur & 17 & 18 \\
Radius at wrist & 31 & 22 \\
Humerus & 30 & 22 \\
Tibia & 1 & 21 \\
\hline
\end{tabular}

A novel outcome of this process was the development of a nurse managed, titrated IV narcotic analgesia policy. The essential elements of this policy are incremental narcotic dosing, recognising that there is wide individual variation in patient's dose requirements for analgesia, ${ }^{12}{ }^{14}$ flexible dose ordering, management of re-assessment of pain and dosing intervals by nursing staff caring for the patient and analgesia ordering by doctors by way of a multi-increment stamp. Nursing staff were chosen for the pivotal role in this approach because of their more frequent contact with patients under their care and because the review and augmentation parts of the policy fitted well into the nursing task of regular observations. It was felt that medical staff were less likely to review patients at frequent, regular intervals or formally review and record pain scores to guide dosing. The policy was implemented in 1995. Copies of the policy and details of nurse education for this role are available from the author on request.

EVALUATION OF THE IMPACT OF THE POLICY

The periods chosen for comparison were 1993 (which was about one year before implementation of the policy) and 1997 (which was about two years after implementation). This latter period was chosen to avoid any "honeymoon" effect of the new policy and to realistically measure sustained change in practice. Acute long bone fractures were chosen as being representative painful conditions for which adequate numbers of cases would be available to provide valid comparisons.

Patients "admitted" to Western Hospital (defined as a stay in the hospital of more than four hours irrespective of the area of treatment) with an admission diagnosis of an acute long bone fracture for the years 1993 and 1997 were identified by the Medical Records Department using a computerised data management system.

A randomly selected subset of available records, matched for patient number, underwent explicit review by a trained research assistant. The data collected included demographic details of the patient, the type, route and amount of analgesia administered during the ED phase of patient management and the type of fracture sustained.

Table 2 Comparison of analgesia administered in 1993 and 1997 for acute long bone fractures

\begin{tabular}{lccl}
\hline Method of analgesia & $1993(\%)$ & $1997(\%)$ & p value $\left(\chi^{2}\right)$ \\
\hline Nil recorded & $14(18)$ & $21(26)$ & \\
Oral only & $13(16)$ & $11(13)$ & \\
IM narcotic only & $42(52)$ & $4(5)$ & $\mathrm{p}<0.001(43.396)$ \\
Combined IM and IV narcotic & $3(4)$ & $1(1)$ & \\
IV narcotic & $7(9)$ & $45(54)$ & $\mathrm{p}<0.001(36.699)$ \\
Femoral nerve block alone & $1(1)$ & $1(1)$ & \\
\hline
\end{tabular}

Data were analysed using $\chi^{2}$ tests of difference in proportions. Data analysis was conducted by the Department of Mathematics (Statistical Consulting Service), Monash University.

\section{Results}

The records of 162 patients were reviewed. Seventy nine of these patients were treated in 1993 and 83 in 1997. The groups were comparable for age and sex. Table 1 shows the types of fractures sustained by the study subjects. The radius, femur and humerus sub-groups are well matched. The discrepancy between the tibia sub-groups is attributable to chance in the availability and selection of records.

In 1993, 53\% of patients with long bone fractures received IM narcotic analgesia compared with $5 \%$ in 1997 . In contrast, in 1993, $6 \%$ of the patients received IV narcotic analgesia compared with $54 \%$ in 1997 . Both these changes are significant $\left(\mathrm{p}<0.001, \chi^{2}\right)$. Table 2 shows the comparison of route and type of analgesia.

\section{Discussion}

One of the primary goals of emergency physicians is the prompt, effective alleviation of pain. Historically, attempts to achieve this aim have been poor both in provision of analgesia and delay to analgesia. ${ }^{3-7}$ No papers have been published assessing the quality of analgesia delivered in Australasian emergency departments.

One method suggested to tackle this problem has been the use of pain management protocols, in particular protocols that favour the use of titrated IV doses of narcotics for acute severe pain. ${ }^{81011}$ Such a protocol for management of pain in an ED was reported by Goodacre and Roden. ${ }^{7}$ However, a number of concerns about such protocols have been raised. They may be broadly classified as process issues concerned with the implementation, utilisation and sustainability of the protocol and safety issues concerned with the potential for adverse events, in particular respiratory depression and cardiovascular instability.

The study ED took a comprehensive process approach to identify issues contributing to inadequate analgesia additional to route of administration and dosing and to improve utilisation and sustainability. This study describes these issues, in particular the incorporation of pain measurement into "routine" observations and a novel approach to the delivery and augmentation of narcotic agents. It demonstrates the effectiveness of the policy by showing a major and sustained change in analgesia ordering and administration practice from IM to titrated IV narcotic for long bone fractures. The finding that $54 \%$ of long bone fractures in the 1997 sample group were treated with IV narcotic compares favourably with the impact of the analgesia protocol by Goodacre and Roden, ${ }^{7}$ which resulted in $37 \%$ of similar patients receiving IV analgesia. It is even more significant as the follow up audit period in Goodacre's study was much closer to the implementation of the protocol than is the case 
in this study and thus is likely to have benefited from a "honeymoon" effect (because of educational and promotional activities related to implementation). In the study ED, the changes in practice have been maintained over more than two years. This durability contrasts with the reported experience of other management protocols. ${ }^{13}$ It is potentially attributable to a number of factors including the example of senior staff and the simplicity and flexibility of the policy such that it has become an integral part of everyday practice.

Although this paper specifically considers the process concerns related to a pain management protocol, the safety issues have also been studied and are reported elsewhere. ${ }^{15}$ In 401 cases audited, there were no cases of respiratory depresssion identified. There were 17 cases of hypotension (blood pressure less than $100 \mathrm{~mm} \mathrm{Hg}$ ), one hypersensitivity reaction and one vasovagal reaction.

Despite its dramatic results, this study has some limitations that must be taken into account when evaluating the results. Although a change in practice is clearly demonstrated, this study does not consider the timeliness or adequacy of analgesia. Although reasonable quality data on pain scores are available for the 1997 cases, no such data are available for 1993. Consideration was given to continuing with previous practice and collecting pain score data before the implementation of the protocol, however this was considered to be unethical. This study is also subject to the general limitations of retrospective studies such as documentation errors and note interpretation. Attempts were made to minimise these by the use of an explicit data retrieval system. The generalisability of this result to other painful conditions might be questioned. The policy was developed for and is applied to all acutely painful conditions so this is unlikely. Similar changes in practice with respect to renal colic have also been shown. ${ }^{16}$

\section{Conclusion}

This project shows that a process approach to improving pain management that resulted in both changes to drug administration and pain assessment and management processes can make a significant and sustained change to analgesia administration practices for patients with long bone fractures in an ED.

I would like to acknowledge the assistance of Ms Justine Neilson, research assistant who undertook the data collection and of the Department of Mathematics (Statistical Consulting Service), Monash University for help with data analysis.

Contributor

Anne-Maree Kelly initiated the study, formulated the hypothesis tested, designed the study methodology (in particular data collection forms), oversaw data collection by the research assistant and wrote the paper.

Funding: this study received funding from the Department of Emergency Medicine departmental funds.

Conflicts of interest: none.

1 Paris PM, Stewart RD, eds. Pain management in emergency medicine. Norwalk, CT: Appelton and Lange, 1988.

2 Walsh M. Pain and anxiety in A\&E attenders. Nursing Standard 1993;7:40-2

3 Wilson JE, Pendleton JM. Oligoanalgesia in the emergency department. Am f Emerg Med 1989;7:620-3.

4 Selbst SM, Clark M. Analgesic use in the emergency department. Ann Emerg Med 1990;19:1010-13.

5 Lewis LM, Lasater LC, Brooks CB. Are emergency physicians too stingy with analgesics? South Med $\mathcal{F}$ 1994;87:7.

6 Ducharme J, Barber C. A prospective blinded study of emergency pain assessment and therapy. $f$ Emerg Med 1995;13:571-5.

7 Goodacre SW, Roden RK. A protocol to improve analgesia use in the accident and emergency department. $\mathcal{F}$ Accid Emerg Med 1996;13:177-9.

8 Reichl M, Bodiwala GG. Use of analgesia in severe pain in the accident and emergency department. Arch Emerg Med 1987;4:25-31.

9 Read JV. Perceptions of nurses and physicians regarding pain management of pediatric emergency room patients. Pediatric Nursing 1994;20:314-18.

10 Acute pain management guideline panel : Acute pain management: Operative or medical procedures and trauma: management: Operative or medical procedures and trauma: of Health and Human Services, 1992 .

11 Ducharme J. Emergency pain management: A Canadian Association of Emergency Physicians consensus document. 7 Emerg Med 1994;12:855-66.

12 Austin KL, Stapleton JV, Mather LE. Multiple intramuscular injections: A major source of variability in analgesic response to meperidine. Pain 1980;8:47-62.

13 Bezerra JA, Strathos TH, Duncan B, et al. Treatment of infants with acute diarrhea: What's recommended and infants with acute diarrhea: What's recc

14 Ginifer C, Kelly AM. Administration of rectal indomethacin does not reduce the requirement for intravenous narcotic analgesia in acute renal colic. Eur f Emerg Med 1996;3: $92-5$

15 Coman M, Kelly AM. Safety of a nurse-managed, titrated analgesia protocol for the management of severe pain in the emergency department. Emerg Med 1999;11:128-31.

16 Kelly AM. Impact of an integrated pain management policy on the management of pain from renal colic in the emergency department. Australian Health Review (in press). 